\title{
Estudio computacional conformacional, espectroscópico, ONL, HOMO-LUMO y reactividad de 1,3,5-trifenilpirazol
}

\author{
Conformational, spectroscopic, NLO, HOMO-LUMO and \\ reactivity of 1,3,5-triphenylpirazole computational study
}

\author{
Estudo conformacional através de simulação \\ computacional, espectroscópico, ONL, HOMO- \\ LUMO e reactividade de 1,3,5-trifenilpirazol
}

\author{
Edgard Fabián Blanco-Acuña ${ }^{1}$; Liddier Pérez-Hincapié ${ }^{1}$; Alfredo Pérez-Gamboa ${ }^{1 *}$ \\ Grey Castellar-Ortega2; María Cely-Bautista²
}

${ }^{1}$ Programa de Química, Universidad del Atlántico, km 7 Vía a Puerto Colombia, Barranquilla-Colombia. ${ }^{2}$ Facultad de Ingeniería, Universidad Autónoma del Caribe, calle 90 n. ${ }^{\circ}$ 46-112, Barranquilla-Colombia.

alfredoperez@mail.uniatlantico.edu.co

Fecha recepción: 29 de mayo de 2017 Fecha aceptación: 10 de septiembre de 2018

\begin{abstract}
Resumen
Los parámetros estructurales de 1,3,5-trifenilpirazol se determinaron con DFT/cam-B3LYP con el conjunto de bases $6-311++G(d, p)$. Los resultados de la estructura molecular optimizada se presentan y comparan con los datos disponibles de rayos $X$ de la molécula o moléculas muy similares. Se proporciona un análisis completo de los espectros observados de las mediciones espectrales de FT-IR, RMN $\left({ }^{1} \mathrm{H}\right.$ y $\left.{ }^{13} \mathrm{C}\right)$ y absorción UV-Vis con TD-DFT en la misma función y conjunto de bases. Los descriptores de reactividad global y local han sido determinados. Las propiedades ONL de esta molécula también fueron investigadas. Las distribuciones de cargas del análisis de poblaciones naturales y el mapa de potencial electrostático están correlacionados. Los resultados calculados y los hallazgos experimentales se discuten y se correlacionan.
\end{abstract}

Palabras clave: química computacional, teoría del funcional de la densidad (DFT), 1,3,5-trifenilpirazol, óptica no lineal, orbitales HOMO-LUMO, descriptores de la reactividad

\begin{abstract}
The structural parameters of 1,3,5-triphenylpyrazole were determined with DFT/cam-B3LYP with the base set $6-311++G(d, p)$. The results of the optimized molecular structure are presented and compared with the available X-ray data of the molecule or very similar molecules. A complete analysis of the observed spectra of the spectral measurements of FT-IR, NMR $\left({ }^{1} \mathrm{H}\right.$ and $\left.{ }^{13} \mathrm{C}\right)$ and UV-Vis absorption with TD-DFT with the same function and set of bases is provided. The descriptors of global and local reactivity have been determined. The NLO properties of this molecule were also investigated. The distributions of NPA and MEP loads are correlated. The calculated results and the experimental findings are discussed and correlated.
\end{abstract}

Keywords: computational chemistry, density functional theory (DFT), 1,3,5-triphenylpyrazole, nonlinear optics, HOMO-LUMO orbitals, reactivity descriptors.

Resumo

Cita: Blanco-Acuña EF, Pérez-Hincapié L, Pérez-Gamboa A, Castellar-Ortega G, Cely-Bautista M. Estudio computacional conformacional, espectroscópico, ONL, HOMO-LUMO y reactividad de 1,3,5-trifenilpirazol. rev.ion. 2018;31(2):51-66. doi:10.18273/revion.v31n2-2018004 
Os parâmetros estruturais do 1,3,5-trifenilpirazole foram determinados com DFT/cam-B3LYP com o conjunto de bases $6-311++G(d, p)$. Os resultados da estrutura molecular otimizada são apresentados e comparados com os dados de raios-X disponíveis da molécula ou moléculas muito semelhantes. Uma análise completa dos espectros observados das medições espectrais de FT-IR, RMN $\left({ }^{1} \mathrm{He}^{13} \mathrm{C}\right)$ e absorção UV-Vis com TD-DFT na mesma função e conjunto de bases é fornecida. As propriedades NLO desta molécula também foram investigadas. As distribuições de cargas NPA e MEP estão correlacionadas. Os resultados calculados e os resultados experimentais são discutidos e correlacionados.

Palavras-chave: química computacional, teoria funcional de densidade (DFT), 1,3,5-trifenilpirazol, ótica não linear, orbitais HOMO-LUMO, descritores de reatividade.

\section{Introducción}

En los últimos años, parte de la investigación química ha mostrado gran interés en el desarrollo de nuevos materiales con aplicaciones electrónicas y ópticas, desde transistores moleculares hasta celdas solares [1,2]. Los compuestos orgánicos con gran número de insaturaciones conjugadas y sistemas aromáticos han llamado la atención en el diseño de moléculas candidatas para materiales en estos dispositivos. Los métodos de la química computacional han demostrado ser una herramienta poderosa y eficiente para la evaluación de las propiedades moleculares y electrónicas de los posibles candidatos a materiales para optoelectrónica [3-5]. La comparación de la fuerza donadora y aceptora de densidad electrónica de los sustituyentes, grado de conjugación, propiedades espectroscópicas y de óptica no lineal permiten el establecimiento de relaciones de estructura-propiedad que facilitan la exploración virtual de muchas moléculas $[6,7]$. Este enfoque es importante en el campo tecnológico y ambiental, debido al ahorro de tiempo y recursos en la exploración en la diversidad molecular, además de los retos que puede representar la síntesis de esos candidatos.

Los derivados de pirazol se han destacado en este campo, por ejemplo, Gondek reportó el uso de un derivado de pirazol como aceptor en la heteroestructura de una celda solar [8]. Asimismo, Amudha et al. observaron un incremento en la conductividad y eficiencia en la conversión de energía en una celda solar de polimetilmetracrilato y fluoruro de polivinilideno al doparla con pirazol [9]. A pesar de estos hallazgos, hasta donde se tiene conocimiento, no existen trabajos que describan las propiedades moleculares, espectroscópicas (IR, RMN, UV-vis) y electrónicas para este tipo de compuestos. Sobre esta base, en este trabajo se presenta la descripción y caracterización de la estructura molecular y electrónica de 1,3,5-trifenilpirazol. La discusión consiste en una evaluación detallada de los orbitales moleculares de frontera (HOMO, HOMO-1, LUMO y LUMO+1), las propiedades de óptica no lineal (ONL), electronegatividad $(\chi)$, dureza $(\eta)$, suavidad $(S)$, análisis de población natural (NPA), el mapa de potencial electrostático (MEP) y los descriptores de reactividad global y local (funciones de Fukui).

\section{Teoría}

El mapa de potencial electrostático (MEP) es un método de cartografía del potencial electrostático, es decir, muestra la distribución de carga del sistema. Con esta superficie es posible determinar la forma, el tamaño y la orientación del momento dipolar de la molécula, representando un método visual para comprender la polaridad relativa [1012]. Adicionalmente, es posible dilucidar cómo las moléculas interactúan entre sí.

Los orbitales de frontera, HOMO y LUMO, según la teoría de Fukui, determinan la reactividad de una molécula frente a otra especie [13]. Los orbitales HOMO-1 y LUMO+1 se denominan donante y aceptor, son niveles de energía que se encuentran por debajo y por encima de los niveles principales, respectivamente [14].

La polarización molecular obtenida por la perturbación de un campo electromagnético $(F)$ se expresa como una serie de Taylor en potencias de $F$. Cada uno de los coeficientes de la serie representa una respuesta particular del sistema. Truncando la serie en el tercer término, definimos el primero como el momento dipolar $(\mu)$, el segundo se denomina polarizabilidad molecular $(\alpha)$ y el tercero se conoce como primera hiperpolarizabilidad $(\beta)$. Cada uno de estos se representa como un tensor; sin embargo, es posible obtener propiedades que son invariantes al sistema de coordenadas partiendo de los elementos de cada tensor. Estas 
propiedades son susceptibles de ser comparadas con los diferentes experimentos. En este sentido, el momento dipolar total se define como el módulo del vector momento dipolar:

$$
\mu_{t o t}=\sqrt{\left(\mu_{x}^{2}+\mu_{y}^{2}+\mu_{z}^{2}\right)}
$$

La polarizabilidad promedio $\alpha_{\text {ave }}$, se define como un tercio de la traza del tensor de polarizabilidad:

$$
\alpha_{\text {ave }}=\frac{1}{3}\left(\alpha_{x x}+\alpha_{y y}+\alpha_{z z}\right)
$$

En el caso de la primera hiperpolarizabilidad $\beta$, se define un vector $\beta\left(\beta_{\mathrm{v}}\right)$, donde cada componente de este vector se calcula como $\beta_{i}=\beta_{i i i}+\beta_{i j j}+\beta_{i k k}$, $\forall i, j, k=\{x, y, z\}$. En consecuencia, el $\beta_{t o t}$, o módulo de $\beta_{v}$, es:

$$
\beta_{t o t}=\left[\left(\beta_{x x x}+\beta_{x y y}+\beta_{x z z}\right)^{2}+\left(\beta_{y y y}+\beta_{y z z}+\beta_{y x x}\right)^{2}+\left(\beta_{z z z}+\beta_{z x x}+\beta_{z y y}\right)^{2}\right]^{\frac{1}{2}}
$$

Esta última propiedad está asociada con la transferencia de carga resultante del movimiento de la nube de electrones a través de la estructura conjugada $\pi$, la cual va en dirección del grupo donante de electrones al grupo aceptor de electrones [15]. La segunda hiperpolarizabilidad responsable de efectos ONL de tercer orden se calcula utilizando la siguiente expresión:

$$
\gamma_{a v e}=\frac{1}{5}[x x x x+y y y y+z z z z+2(x x y y+x x z z+y y z z)]
$$

Las moléculas con un gran momento dipolar y un medio para cambiar la densidad electrónica tendrán hiperpolarizabilidades grandes. En general se puede afirmar que dicha propiedad depende de la fuerza de los grupos dador y aceptor, así como de la longitud de la conjugación en el sistema que los une, por otra parte, en sistemas con anillos aromáticos sustituidos la planaridad afecta e influye en el tamaño del sistema de electrones $\pi$ y en la movilidad de electrones $[16,17]$.

Por otro lado, propiedades como el potencial químico $(\mu)$ y la dureza $(\eta)$ son expresadas sobre las bases de la aproximación de diferencias finitas en términos de $I$ y $A$ [18]. La suavidad $(S)$ es un parámetro recíproco de la dureza, $(\chi)$ corresponde a la electronegatividad $(\omega)$, equivale al índice de electrofilicidad; dichos parámetros se expresan en las siguientes ecuaciones:

$$
\begin{gathered}
\boldsymbol{\mu}=\frac{1}{2}(\boldsymbol{I}+\boldsymbol{A})=-\chi \\
\boldsymbol{\eta}=\frac{1}{2}(\boldsymbol{I}-\boldsymbol{A}) \\
\boldsymbol{S}=\frac{1}{\eta} \\
\boldsymbol{\omega}=\frac{\chi^{2}}{2 \boldsymbol{\eta}}
\end{gathered}
$$

La función de Fukui se interpreta como el cambio de potencial químico dada una perturbación externa o la variación de la densidad electrónica, y se da al cambiar su número de electrones, ya que cuando el sistema en estudio recibe o cede electrones se define la capacidad electrofílica o nucleofílica de la especie química. La función de Fukui refleja entonces la reactividad de diferentes sitios dentro de una molécula, es decir, la selectividad, en donde la dirección de ataque preferida por un reactivo será aquella donde la función de Fukui presente los valores más altos [19]. Por otro lado, la suavidad local se conoce como un indicador para las secuencias de reactividad intermolecular, dado que la suavidad local es una cantidad producto de un descriptor de reactividad global [19].

\section{Metodología}

Se utilizó el software Gaussian09 revisión A02 [20] para llevar a cabo los cálculos químico-cuánticos y GaussView 5.08 [21] como visualizador. La optimización de la geometría se realizó al nivel DFT/cam-B-3LYP/6-311++G(d,p) [22] sin restricciones de simetría, y se confirmó la ausencia de autovalores negativos de la matriz hessiana mediante el análisis vibracional. La frecuencia de las vibraciones del espectro IR teórico se corrigieron con un factor de escala de 0,9461 [2325]. Con esta estructura se realizaron cálculos de 
energía en conjunto con un análisis de población natural (NPA) para la molécula neutra e ionizada (positiva y negativa) para determinar los centros nucleofílicos, electrofílicos y radicalarios, y obtener posteriormente los descriptores de la reactividad local y las funciones de Fukui nucleofílica y electrofílica condensadas. El espectro UV-Vis y los orbitales de frontera HOMO-1, HOMO, LUMO y LUMO+1 se obtuvieron a partir del cálculo DFT dependiente del tiempo (TDDFT). Las propiedades ópticas lineales y no lineales estáticas, momentos dipolares $(\mu)$, polarizabilidad $(\alpha)$, hiperpolarizabilidad de primer orden $(\beta)$ e hiperpolarizabilidad de segundo orden $(\gamma)$ se obtuvieron mediante DFT con perturbaciones acopladas (CPDFT). Las ecuaciones (1), (2), (3) y (4) se emplearon para el cálculo de estos parámetros. Los espectros de $\mathrm{RMN}$ de ${ }^{1} \mathrm{H}$ y ${ }^{13} \mathrm{C}$ se obtuvieron mediante GIAO, sin considerar acoplamientos spin-spin. Adicionalmente, los desplazamientos se calcularon también con los programas ChemBioDraw [26] y MestReNova [27].

\section{Resultados y discusión}

\section{Geometría molecular}

La numeración de los átomos diferentes a hidrógeno usada en la estructura modelada se muestra en la figura 1.

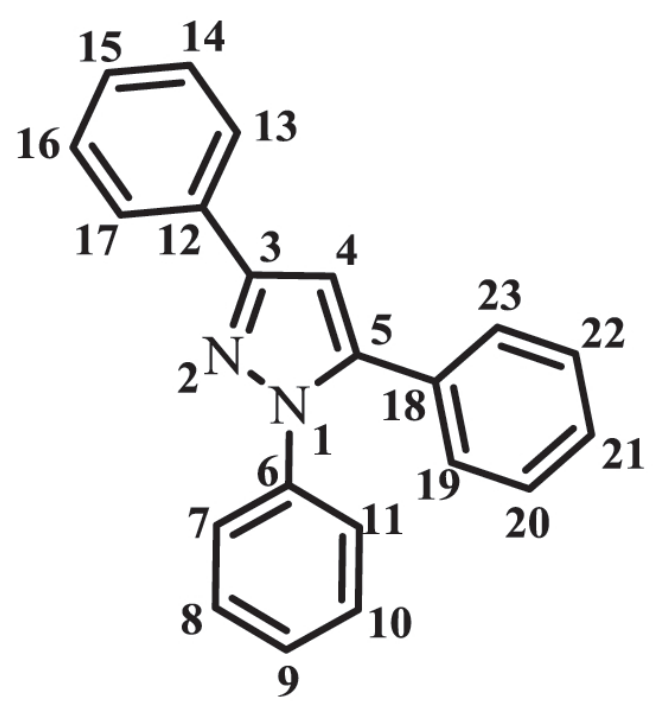

Figura 1. Numeración de la estructura molecular 1,3,5-trifenilpirazol.

Se conoce que las longitudes de enlace $\mathrm{C}-\mathrm{C}$ y $\mathrm{C}-\mathrm{H}$ en el benceno son 1,40 y 1,08 , respectivamente [28]. Tomando estos valores como referencia se observa en la tabla 1 que los tres fenilos sustituyentes conservan sus distancias de enlace sin verse modificadas por el anillo de pirazol. Asimismo, se esperaría una modificación en los enlaces de los fenilos sustituyentes en las posiciones 1 y 5 del pirazol, debido a su gran cercanía, pero esto no se presenta, ya que la molécula, al adquirir su geometría de mínima energía, mantiene los parámetros de las longitudes de enlaces.

El anillo de pirazol debería ser un pentágono regular con ángulos de $108^{\circ}$, pero debido a la presencia de los dos nitrógenos esta geometría se modifica muy levemente en el ángulo C3C4-C5 y toma un valor de $106,2^{\circ}$, que es menor al esperado. Comparando los valores experimentales con los calculados se observa que estos últimos presentan mayor distorsión de un pentágono regular, pero aún son muy cercanos. Con respecto a los ángulos de los fenilos sustituyentes y su relación con el anillo de pirazol se observan valores muy cercanos a los esperados de $120^{\circ}$ para un hexágono regular. Esto se respalda con los valores experimentales que son muy cercanos a los calculados. 
Tabla 1. Longitudes de enlaces calculadas y descritas para el compuesto 1,3,5-trifenilpirazol.

\begin{tabular}{|c|c|c|c|}
\hline Núcleo & $\begin{array}{l}\text { Átomos } \\
\text { enlazados }\end{array}$ & $\begin{array}{l}\text { Longitud de enlace } \\
\text { calculada }(\AA)\end{array}$ & $\begin{array}{l}\text { Longitud de enlace } \\
\text { experimental }(\hat{A})\end{array}$ \\
\hline \multirow[t]{8}{*}{ Pirazol } & N1 - N2 & 1,348 & 1,354 \\
\hline & N1 - C5 & 1,369 & 1,343 \\
\hline & N1 - C6 & 1,423 & \\
\hline & N2 - C3 & 1,327 & 1,350 \\
\hline & C3 - C4 & 1,414 & 1,376 \\
\hline & C3 - C12 & 1,473 & 1,473 \\
\hline & C4 - C5 & 1,377 & 1,388 \\
\hline & C5 - C18 & 1,474 & 1,473 \\
\hline \multirow[t]{6}{*}{ 1-Fenil } & C6 - C7 & 1,392 & \\
\hline & C6 - C11 & 1,391 & \\
\hline & C7 - C8 & 1,389 & \\
\hline & C8 - C9 & 1,392 & \\
\hline & C9 - C10 & 1,391 & \\
\hline & C10 - C11 & 1,390 & \\
\hline \multirow[t]{6}{*}{ 3-Fenil } & C12 - C13 & 1,397 & \\
\hline & C12 - C17 & 1,399 & \\
\hline & C13 - C14 & 1,390 & \\
\hline & C14 - C15 & 1,391 & \\
\hline & C15 - C16 & 1,393 & \\
\hline & C16 - C17 & 1,388 & \\
\hline \multirow[t]{6}{*}{ 5-Fenil } & C18 - C19 & 1,398 & \\
\hline & C18 - C23 & 1,398 & \\
\hline & C19 - C20 & 1,389 & \\
\hline & C20 - C21 & 1,392 & \\
\hline & C21 - C22 & 1,391 & \\
\hline & $\mathrm{C} 22-\mathrm{C} 23$ & 1,391 & \\
\hline
\end{tabular}

Tabla 2. Ángulos de enlaces calculados para el compuesto.

\begin{tabular}{|c|c|c|c|c|c|}
\hline $\begin{array}{l}\text { Átomos } \\
\text { enlazados }\end{array}$ & $\begin{array}{c}\text { Calculados } \\
\left(^{\circ}\right)\end{array}$ & $\begin{array}{c}\text { Experimental } \\
\left({ }^{\circ}\right)\end{array}$ & $\begin{array}{l}\text { Átomos } \\
\text { enlazados }\end{array}$ & $\begin{array}{c}\text { Calculados } \\
\left(^{\circ}\right)\end{array}$ & $\begin{array}{c}\text { Experimental } \\
\left({ }^{\circ}\right)\end{array}$ \\
\hline $\mathrm{N} 2-\mathrm{N} 1-\mathrm{C} 5$ & 111,8 & 108,5 & C3 - C12-C13 & 120,8 & 121,4 \\
\hline N2 - N1 - C6 & 118,4 & & C3 - C12 - C17 & 120,4 & 118,6 \\
\hline C5 - N1 - C6 & 129,5 & & $\mathrm{C} 13-\mathrm{C} 12-\mathrm{C} 17$ & 118,7 & \\
\hline $\mathrm{N} 1-\mathrm{N} 2-\mathrm{C} 3$ & 106,0 & 108,6 & C12 - C13 - C14 & 120,7 & \\
\hline $\mathrm{N} 2-\mathrm{C} 3-\mathrm{C} 4$ & 110,4 & 108,3 & C13 - C14 - C15 & 120,1 & \\
\hline N2 - C3 - C12 & 120,8 & 121,1 & C14 - C15 - C16 & 119,4 & \\
\hline C4 - C3 - C12 & 128,7 & 130,6 & C15 - C16 - C17 & 120,3 & \\
\hline C3 - C4 - C5 & 105,6 & 106,2 & C12 - C17 - C16 & 120,5 & \\
\hline N1 - C5 - C4 & 105,9 & 108,3 & & & \\
\hline N1 - C5 - C18 & 125,4 & 121,1 & C5 - C18 - C19 & 121,5 & 120,7 \\
\hline C4 - C5 - C18 & 128,5 & 130,5 & C5 - C18 - C23 & 119.4 & 119,3 \\
\hline N1 - C6 - C7 & 118,8 & & C19 - C18 - C23 & 120,4 & \\
\hline \multirow[t]{2}{*}{ N1 - C6 - C11 } & 120,5 & & C18 - C19 - C20 & 120,2 & \\
\hline & & & C19 - C20 - C21 & 119,6 & \\
\hline C7 - C6 - C11 & 120,5 & & $\mathrm{C} 20-\mathrm{C} 21-\mathrm{C} 22$ & 120,1 & \\
\hline $\mathrm{C} 6-\mathrm{C} 7-\mathrm{C} 8$ & 119,4 & & C21 - C22 - C23 & 120,5 & \\
\hline C7 - C8 - C9 & 120,3 & & C18 - C23 - C22 & 121,5 & \\
\hline C8 - C9 - C10 & 119,7 & & & & \\
\hline C9 - C10 - C11 & 120,3 & & & & \\
\hline C6 - C11 - C10 & 119,4 & & & & \\
\hline
\end{tabular}


Los valores de los ángulos diedros del pirazol cercanos a cero grados demuestran la planaridad del anillo. Este comportamiento también lo presentan internamente los fenilos que sustituyen el pirazol. La disposición espacial de los fenilos con respecto al pirazol no es coplanar para los 1-Ph (C5-N1-C6-C7= 137, $8^{\circ}$ y 5-Ph (C4-C5-C18-C19= $129,4^{\circ}$. Sólo el 3-Ph (N2-C3-C12-C13=179,9 presenta coplanaridad con el pirazol (ver figura 2).

Tabla 3. Ángulos diedros de enlaces calculados.

\begin{tabular}{|c|c|c|c|c|c|}
\hline $\begin{array}{l}\text { Átomos } \\
\text { enlazados }\end{array}$ & $\begin{array}{c}\text { Calculados } \\
\left({ }^{\circ}\right)\end{array}$ & $\begin{array}{c}\text { Átomos } \\
\text { enlazados }\end{array}$ & $\begin{array}{c}\text { Calculados } \\
\left({ }^{\circ}\right)\end{array}$ & $\begin{array}{l}\text { Átomos } \\
\text { enlazados }\end{array}$ & $\begin{array}{c}\text { Calculados } \\
\left({ }^{\circ}\right)\end{array}$ \\
\hline C5-N1-N2-C3 & 0,3 & N1-N2-C3-C12 & 179,9 & C4-C5-C18-C19 & 129,4 \\
\hline N2-N1-C5-C4 & $-0,5$ & C12-C3-C4-C5 & 179,6 & $\mathrm{C} 4-\mathrm{C} 5-\mathrm{C} 18-\mathrm{C} 23$ & $-47,8$ \\
\hline N1-N2-C3-C4 & 0,0 & N2-C3-C12-C13 & 179,9 & C5-C18-C19-C20 & $-177,6$ \\
\hline N2-C3-C4-C5 & $-0,3$ & N2-C3-C12-C17 & $-0,1$ & C23-C18-C19-C20 & $-0,3$ \\
\hline \multirow[t]{2}{*}{ C3-C4-C5-N1 } & 0,5 & C4-C3-C12-C13 & $-0,1$ & C5-C18-C23-C22 & 177,4 \\
\hline & & C4-C3-C12-C17 & 179,7 & C19-C18-C23-C22 & 0,2 \\
\hline C6-N1-N2-C3 & $-176,5$ & C3-C12-C13-C14 & 179,9 & C18-C19-C20-C21 & 0,3 \\
\hline C6-N1-C5-C4 & 175,8 & C17-C12-C13-C14 & 0,0 & C19-C20-C21-C22 & 0,1 \\
\hline N1-C6-C7-C8 & 179,0 & C3-C12-C17-C16 & $-179,9$ & $\mathrm{C} 20-\mathrm{C} 21-\mathrm{C} 22-\mathrm{C} 23$ & $-0,3$ \\
\hline N2-N1-C6-C7 & $-45,9$ & C13-C12-C17-C16 & 0,0 & $\mathrm{C} 21-\mathrm{C} 22-\mathrm{C} 23-\mathrm{C} 18$ & 0,2 \\
\hline N2-N1-C6-C11 & 132,5 & C12-C13-C14-C15 & 0,0 & C3-C4-C5-C18 & $-176,0$ \\
\hline C5-N1-C6-C7 & 137,9 & C13-C14-C15-C16 & 0,0 & N1-C5-C18-C19 & $-46,5$ \\
\hline C5-N1-C6-C11 & $-43,6$ & C14-C15-C16-C17 & 0,0 & N1-C5-C18-C23 & 136,2 \\
\hline C11-C6-C7-C8 & 0,6 & $\mathrm{C} 15-\mathrm{C} 16-\mathrm{C} 17-\mathrm{C} 12$ & 0,0 & C7-C8-C9-C10 & $-0,6$ \\
\hline N1-C6-C11-C10 & $-179,7$ & & & C8-C9-C10-C11 & 0,0 \\
\hline C7-C6-C11-C10 & $-1,2$ & N2-N1-C5-C18 & 176,1 & C9-C10-C11-C6 & 0,9 \\
\hline C6-C7-C8-C9 & 0,4 & C6-N1-C5-C18 & $-7,4$ & & \\
\hline
\end{tabular}
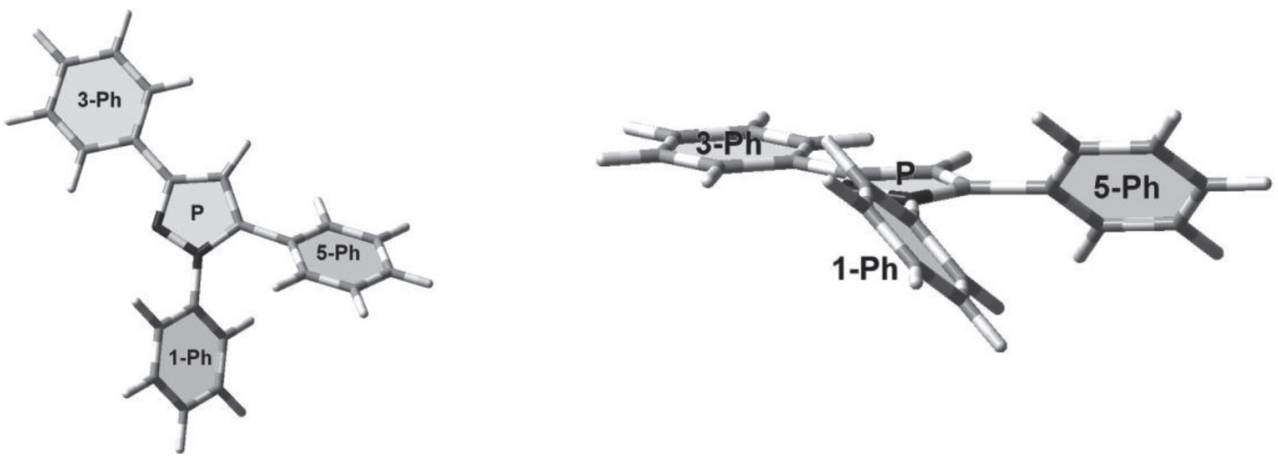

Figura 2. Relación espacial de los planos $\mathrm{P}, 1-\mathrm{Ph}, 3-\mathrm{Ph}$ y 5-Ph en la molécula. 


\section{Espectroscopia vibracional}

El espectro de IR se presenta en la figura 3. Las vibraciones de $\mathrm{C}-\mathrm{H}$ para benceno se observan normalmente en el rango de 3000 a $3100 \mathrm{~cm}^{-1}$ [29] con una intensidad media. Las vibraciones calculadas que se muestran en la tabla 4 se correlacionaron bien con los datos experimentales $\left(r^{2}=0,9985\right)$. Los estiramientos $\mathrm{C}=\mathrm{C}$ del benceno generalmente aparecen entre 1400 a $1600 \mathrm{~cm}^{-1}$ $[30,31]$. En la Tabla 4 se observa que todos los estiramientos experimentales y calculados presentan buena correlación y se encuentran en la región esperada, a excepción de las frecuencias 1363 (1353), 1214 (1283) y 1175 (1190) y 921 (934) que no corresponden a estiramientos en los fenilos y que se atribuyen a las deformaciones y estiramientos del núcleo de pirazol.

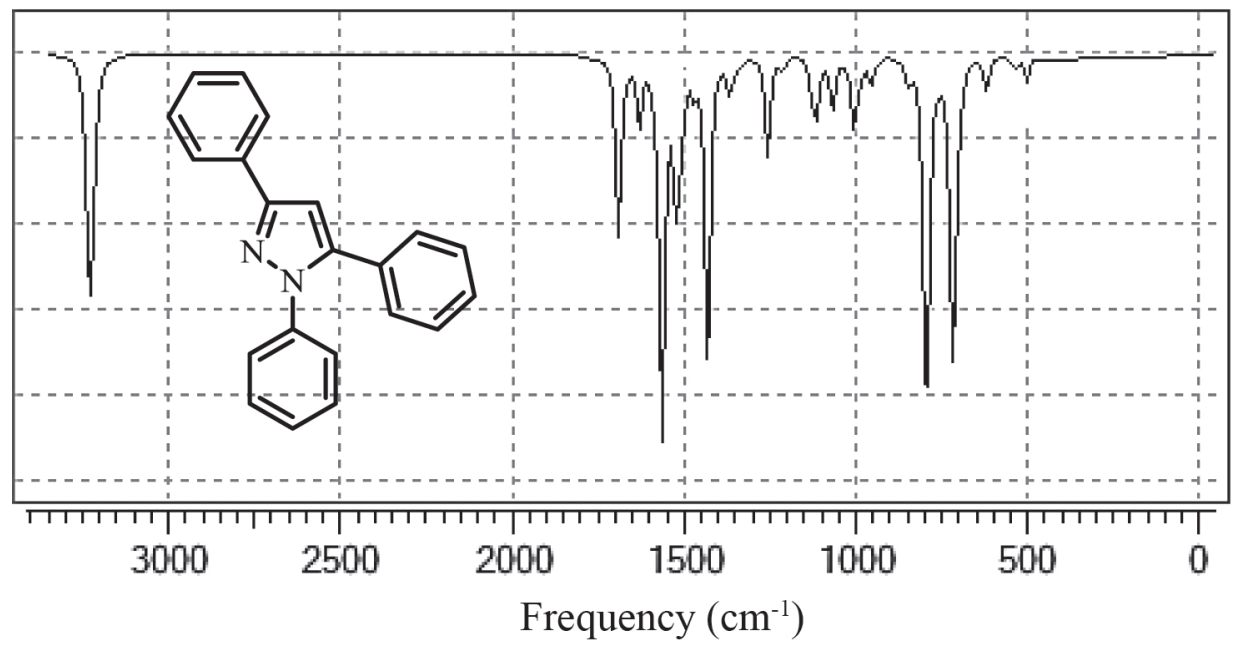

Figura 3. Espectro IR de 1,3,5-trifenilpirazol calculado al nivel DFT/cam-B3LYP/6-311++G(d,p).

Tabla 4. Análisis comparativo de las vibraciones calculadas y experimentales del espectro IR de 1,3,5-trifenilpirazol.

\begin{tabular}{ccccl}
\hline \multicolumn{2}{c}{ Vibración } & Intensidad & Experimental & \\
\cline { 1 - 2 } Calculada & Escalada & & & Asignación \\
3229 & 3055 & Media & 3121 & Estiramiento en el plano C4-H24 \\
1690 & 3048 & Media & 3062 & Estiramientos asimétricos C-H de los fenilo \\
1629 & 1599 & Media & 1596 & Estiramientos simétricos de C=C de los fenilos \\
1564 & 1541 & Baja & 1546 & Deformación aromática y estiramientos varios \\
1524 & 1479 & Fuerte & 1496 & Deformación aromática y estiramientos varios \\
1513 & 1442 & Baja & 1484 & Deformación aromática y estiramientos varios \\
1430 & 1432 & Baja & 1457 & Deformación aromática y estiramientos varios \\
1356 & 1353 & Fuerte & 1363 & Estiramientos asimétricos del pirazol \\
1257 & 1190 & Baja & 1214 & Deformación del pirazol y estiramientos varios. \\
1128 & 1067 & Baja & 1175 & Estiramiento N1-N2 \\
1067 & 1009 & Baja & 1066 & Deformaciones C-H en el plano \\
1005 & 950 & Baja & 1022 & Deformaciones C-H en el plano \\
987 & 934 & Baja & 957 & Deformaciones C-H fuera del plano \\
795 & 752 & Fuerte & 766 & Deformaciones C-H fuera del plano \\
722 & 683 & Media & 701 & Deformaciones C-H fuera del plano \\
\hline
\end{tabular}


Las deformaciones $\mathrm{C}-\mathrm{H}$ en el plano se observan entre 1000 a $1300 \mathrm{~cm}^{-1}$ como bandas débiles [30], por lo cual las dos primeras bandas de la tabla 4 muestran estas vibraciones en los anillos de fenilo. A diferencia de esto, las vibraciones $\mathrm{C}-\mathrm{H}$ fuera del plano se presentan entre 800 a $1000 \mathrm{~cm}^{-1}$ [28], por esto la banda de 957 (950) $\mathrm{cm}^{-1}$ representa este tipo de estiramiento en el fenilo de la posición 3. Asimismo, se presentan dos bandas de 766 (752) y 701 (683) $\mathrm{cm}^{-1}$ que están por debajo de esta región, lo que se atribuye a la cercanía de los fenilos en las posiciones 1 y 5 con los nitrógenos del pirazol. Por último, se observa una banda en
$921(934) \mathrm{cm}^{-1}$ que se atribuye a la deformación en el plano del pirazol, debido a los estiramientos $\mathrm{C} 3=\mathrm{N} 2$ y $\mathrm{C} 3=\mathrm{C} 4$, los cuales producen que la frecuencia de vibración disminuya y no se observe en la región esperada de 1400 a $3100 \mathrm{~cm}^{-1}$ [31].

\section{Resonancia magnética nuclear (RMN)}

Los desplazamientos químicos teóricos de RMN ${ }^{1} \mathrm{H}$ de y $\mathrm{RMN}{ }^{13} \mathrm{C}$ de $1,3,5$-trifenilpirazol junto con los valores experimentales se presentan en las tablas 5 y 6 [32].

Tabla 5. Comparación de los desplazamientos químicos calculados y experimentales de $\mathrm{RMN}{ }^{1} \mathrm{H}$ del compuesto [33,34].

\begin{tabular}{ccccc}
\hline Señal & Gaussian & Chemdraw & MestReNova & Experimental \\
\hline H4 & 7,46 & 7,02 & $7,31(\mathrm{~s}, 1 \mathrm{H})$ & $6,83(\mathrm{~s}, 1 \mathrm{H})$, \\
p-Ph3 & 8,02 & 7,49 & $7,34(\mathrm{~m}, 1 \mathrm{H})$ & \\
p-Ph5 & 7,93 & 7,49 & $7,36(\mathrm{~m}, 1 \mathrm{H})$ & \\
m-Ph3 & $8,08-8,13$ & 7,54 & $7,43(\mathrm{dd}, 2 \mathrm{H})$ & \\
m-Ph5 & $7,93-8,24$ & 7,08 & $7,43(\mathrm{dd}, 2 \mathrm{H})$ & \\
p-Ph1 & 8,10 & 7,52 & $7,47(\mathrm{dd}, 1 \mathrm{H})$ & \\
m-Ph1 & $7,72-8,20$ & 7,60 & $7,52(\mathrm{dd}, 2 \mathrm{H})$ & \\
o-Ph1 & $7,31-8,62$ & 7,60 & $7,59(\mathrm{~d}, 2 \mathrm{H})$ & \\
o-Ph5 & $7,33-8,27$ & 7,08 & $7,64(\mathrm{~d}, 2 \mathrm{H})$ & \\
o-Ph3 & $8,55-9,13$ & 8,06 & $7,65(\mathrm{~d}, 2 \mathrm{H})$ & $7,93(\mathrm{~d}, 2 \mathrm{H})$ \\
\hline
\end{tabular}

Se observa que los valores de $\delta_{\text {cal }}$ y $\delta_{\text {exp }}$ son cercanos entre sí, especialmente con los programas MestReNova y Chemdraw. Un estudio de correlación lineal mostró que $\delta_{\text {exp }}=0,7649 \delta_{\text {cal }}+1,3525$ con un coeficiente de determinación $\left(r^{2}\right)$ igual a 1 . El menor valor de $\delta$ lo presenta $\mathrm{H} 4$, lo cual es coherente con el hecho de que se encuentra rodeado de una gran densidad electrónica proveniente de los fenilos en posiciones 3 y 5 . Además, el anillo de pirazol; por ende, necesitará mayor campo para que su spin entre en resonancia. Caso contrario sucede con $\mathrm{H} 30$ y H31, que se encuentran más alejados de las nubes electrónicas de los fenilos 1 y 5 , por lo que necesita menor campo magnético para entrar en resonancia. En cuanto a los hidrógenos $\mathrm{H} 25$ a H29 y H32 a H39, al tener desplazamientos químicos muy cercanos, producen un acoplamiento fuerte que genera un multiplete que integra para 13 hidrógenos, en el cual no se pueden distinguir las señales de cada hidrógeno. La regresión lineal para los desplazamientos químicos calculados y experimentales de RMN ${ }^{13} \mathrm{C}$ muestra una buena correlación entre estos valores $\left(R^{2}=0,966\right)$.

C3 y C5 presentan los mayores valores de. Esto es de esperarse, debido a que cada uno está enlazado a un átomo de nitrógeno, el cual, al ser más electronegativo que el carbono, atrae su densidad electrónica, y crea una desprotección y en consecuencia el campo aplicado debe ser menor. Caso contrario sucede con $\mathrm{C} 4$, que al estar rodeado por 2 fenilos y la densidad electrónica del pirazol, se necesita un campo aplicado mayor, lo que genera un desplazamiento químico menor. 
rev.ion. 2018;31(2):51-66. Bucaramanga (Colombia)

Tabla 6. Comparación de los desplazamientos químicos calculados y experimentales de $\operatorname{RMN}{ }^{13} \mathrm{C}[35,36]$.

\begin{tabular}{ccccc}
\hline Núcleo & MestReNova & ChemDraw & Gaussian & Experimental \\
\hline C-4 & 106,8 & 106,2 & 89,6 & 105,4 \\
o-C1-Ph & 122,3 & 124,5 & 110,0 & 125,5 \\
o-C1-Ph & 122,3 & 124,5 & 110,5 & 125,5 \\
o-C3-Ph & 126,8 & 127,5 & 109,1 & 125,4 \\
o-C3-Ph & 126,8 & 127,5 & 110,8 & 125,4 \\
o-C5-Ph & 126,8 & 127,5 & 116,4 & 128,6 \\
o-C5-Ph & 126,8 & 127,5 & 116,6 & 128,6 \\
p-C1-Ph & 128,5 & 126,2 & 111,8 & 127,9 \\
m-C3-Ph & 128,5 & 129,2 & 114,1 & 128,7 \\
m-C3-Ph & 128,5 & 129,2 & 114,2 & 128,7 \\
p-C5-Ph & 128,6 & 128,7 & 114,1 & 129,0 \\
p-C3-Ph & 128,6 & 128,7 & 112,5 & 128,2 \\
m-C5-Ph & 129,2 & 129,2 & 111,9 & 128,9 \\
m-C5-Ph & 129,2 & 129,2 & 113,1 & 128,9 \\
m-C1-Ph & 129,3 & 129,3 & 114,2 & 129,2 \\
m-C1-Ph & 129,3 & 129,3 & 115,7 & 129,2 \\
ipso-C5-Ph & 131,4 & 129,9 & 116,9 & 130,1 \\
ipso-C3-Ph & 132,5 & 133,0 & 119,6 & 132,8 \\
ipso-C1-Ph & 139,3 & 139,7 & 127,7 & 139,3 \\
C5 & 142,7 & 144,3 & 132,0 & 137,0 \\
C3 & 152,9 & 151,1 & \\
\hline
\end{tabular}

\section{Espectroscopia electrónica (UV-vis)}

La naturaleza de las transiciones electrónicas, la energía de las transiciones $(E)$, las longitudes de onda de máxima absorción $\left(\lambda_{\max }\right)$ y la fuerza oscilatoria $(f)$ para los estados excitados de la molécula fueron calculadas por varios métodos.
A continuación, se presentan la discusión de los resultados obtenidos (tabla 7 ) para 1,3,5-trifenilpirazol, comparándolos con el dato experimental de $252 \mathrm{~nm}$ [37]. En la tabla 8 se presentan los resultados de un estudio comparativo con otros niveles de cálculo.

Tabla 7. Energía, banda de absorción, fuerza del oscilador y transición electrónica de 1,3,5-trifenilpirazol al nivel TD-DFT/cam-B3LYP/6311++G(dp).

\begin{tabular}{cccclc}
\hline $\begin{array}{c}\text { Estado } \\
\text { excitado }\end{array}$ & $\begin{array}{c}\text { Energía } \\
(\mathbf{e V})\end{array}$ & $\begin{array}{c}\text { Longitud máxima } \\
\left(\lambda_{\max }\right)\end{array}$ & $\begin{array}{c}\text { Fuerza del } \\
\text { oscilador }(\boldsymbol{f})\end{array}$ & \multicolumn{2}{c}{$\begin{array}{c}\text { Transición } \\
\text { electrónica }\end{array}$} \\
\hline 1 & 4,8029 & 258,15 & 0,3576 & HOMO $\rightarrow$ LUMO & 0,64041 \\
2 & 5,1302 & 241,68 & 0,2072 & HOMO $\rightarrow$ LUMO+2 & 0,38092 \\
3 & 5,1567 & 240,43 & 0,0188 & HOMO $\rightarrow$ LUMO+3 & 0,31036 \\
4 & 5,2514 & 236,10 & 0,5466 & HOMO $\rightarrow$ LUMO+2 & 0,37014 \\
5 & 5,2761 & 234,99 & 0,0036 & HOMO-6 $\rightarrow$ LUMO & 0,29487 \\
6 & 5,3890 & 230,07 & 0,0900 & HOMO-1 $\rightarrow$ LUMO & 0,55053 \\
7 & 5,7346 & 216,20 & 0,0217 & HOMO $\rightarrow$ LUMO+6 & 0,39861 \\
8 & 5,7639 & 215,11 & 0,0157 & HOMO $\rightarrow$ LUMO+1 & 0,36298 \\
9 & 5,9204 & 209,42 & 0,0363 & HOMO-1 $\rightarrow$ LUMO+2 & 0,50593 \\
10 & 5,9444 & 208,57 & 0,0192 & HOMO $\rightarrow$ LUMO+5 & 0,34087 \\
\hline
\end{tabular}


Tabla 8. Comparación de los resultados obtenidos en fase gas de cálculos UV-vis con la longitud de onda " $\lambda " \delta$ $\left(\lambda_{\max }\right)$ experimental.

\begin{tabular}{lccccc}
\hline \multicolumn{1}{c}{ Método } & $\boldsymbol{E}(\mathbf{e V})$ & $\boldsymbol{f}$ & $\boldsymbol{\lambda}_{\max }(\mathbf{n m})$ & \multicolumn{1}{c}{ Asignación } \\
\hline DFT/cam-B3LYP/6311++G(dp) & 5,2514 & 0,5466 & 236,10 & $\mathrm{H} \rightarrow \mathrm{L}+2$ & 0,37014 \\
DFT/cam-B3LYP/631g(2d) & 5,3899 & 0,7826 & 230,03 & $\mathrm{H}-1 \rightarrow \mathrm{L}$ & 0,42906 \\
DFT/B3LYP/6-31+G(d,p) & 4,5503 & 0,2134 & 272,67 & $\mathrm{H} \rightarrow \mathrm{L}+1$ & 0,53917 \\
M06-2X/6-31G(2d) & & & & & \\
ZINDO & 3,9034 & 0,7892 & 317,63 & $\mathrm{H} \rightarrow \mathrm{L}$ & 0,60205 \\
\hline
\end{tabular}

\section{Superficie de energía potencial (MEP)}

En la figura 4 se ilustran los sitios reactivos de la molécula sobre el anillo 3-Ph y los nitrógenos del pirazol con distribución de carga con los valores de potencial de $-5.101 \times 10^{-2}$ a $5.101 \times 10^{-2}$. En el resto de la molécula se muestra un color gris tenue que indica que la distribución de electrones en esta zona es neutra o intermedia, es decir, que existe un balance de la densidad electrónica, el cual se da por la presencia de enlaces.

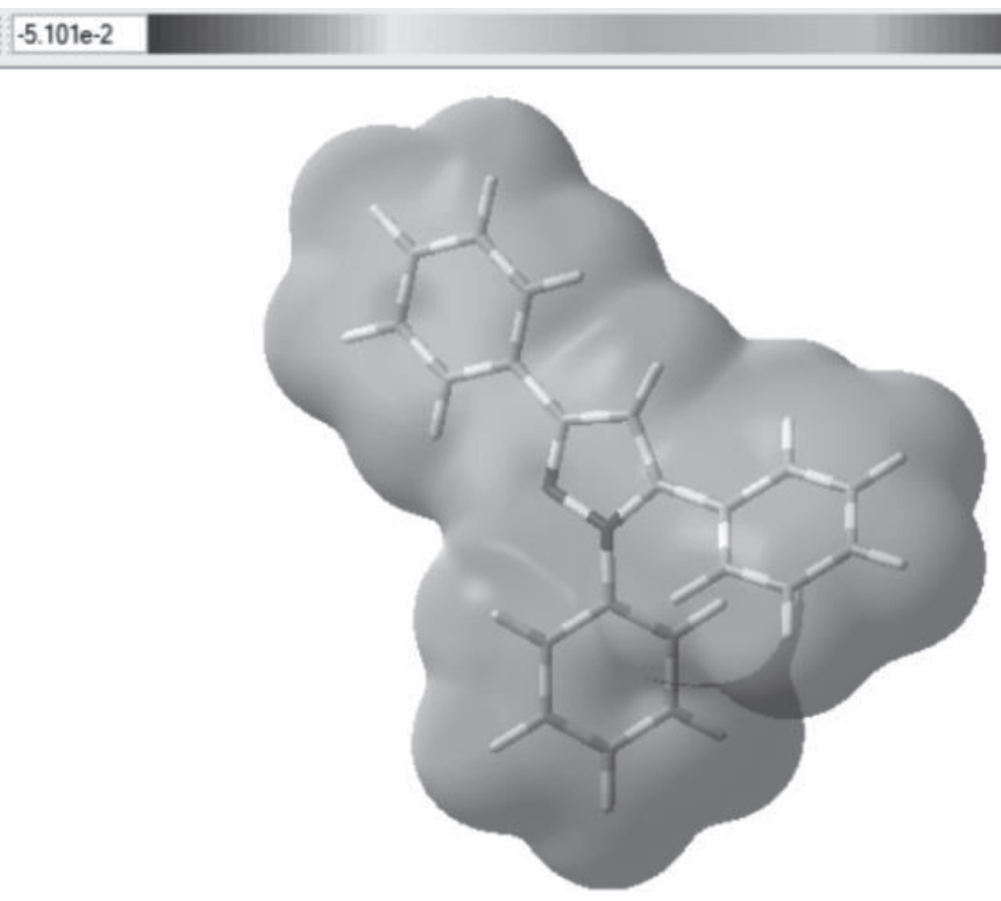

Figura 4. Mapa de potencial electrostático para 1,3,5-trifenilpirazol.

\section{Orbitales de frontera}

Los valores calculados de energía de los orbitales frontera HOMO-1, HOMO, LUMO y LUMO+1 y band-gap se encuentran tabulados en la tabla 9. La figura 5 muestra que la mayor densidad electrónica de los orbitales HOMO se concentra en la región del anillo de pirazol y el fenilo en posición 3, es decir, en los anillos coplanares, ya que se carece de sustituyentes. Por otro lado, en el orbital LUMO la densidad electrónica está distribuida sobre todo el sistema, pero en especial en la región del pirazol, los fenilos en posiciones 1 y 5 , siendo así el orbital HOMO el dador y el orbital LUMO el aceptor. En el HOMO -1 la densidad electrónica se centra en la parte derecha de todo el sistema, es decir, dicha densidad se encuentra concentrada sobre el fenilo en posición 5, por otra parte, en el LUMO +1 la densidad electrónica se encuentra totalmente concentrada en los fragmentos fenilo 3 y 5 . 


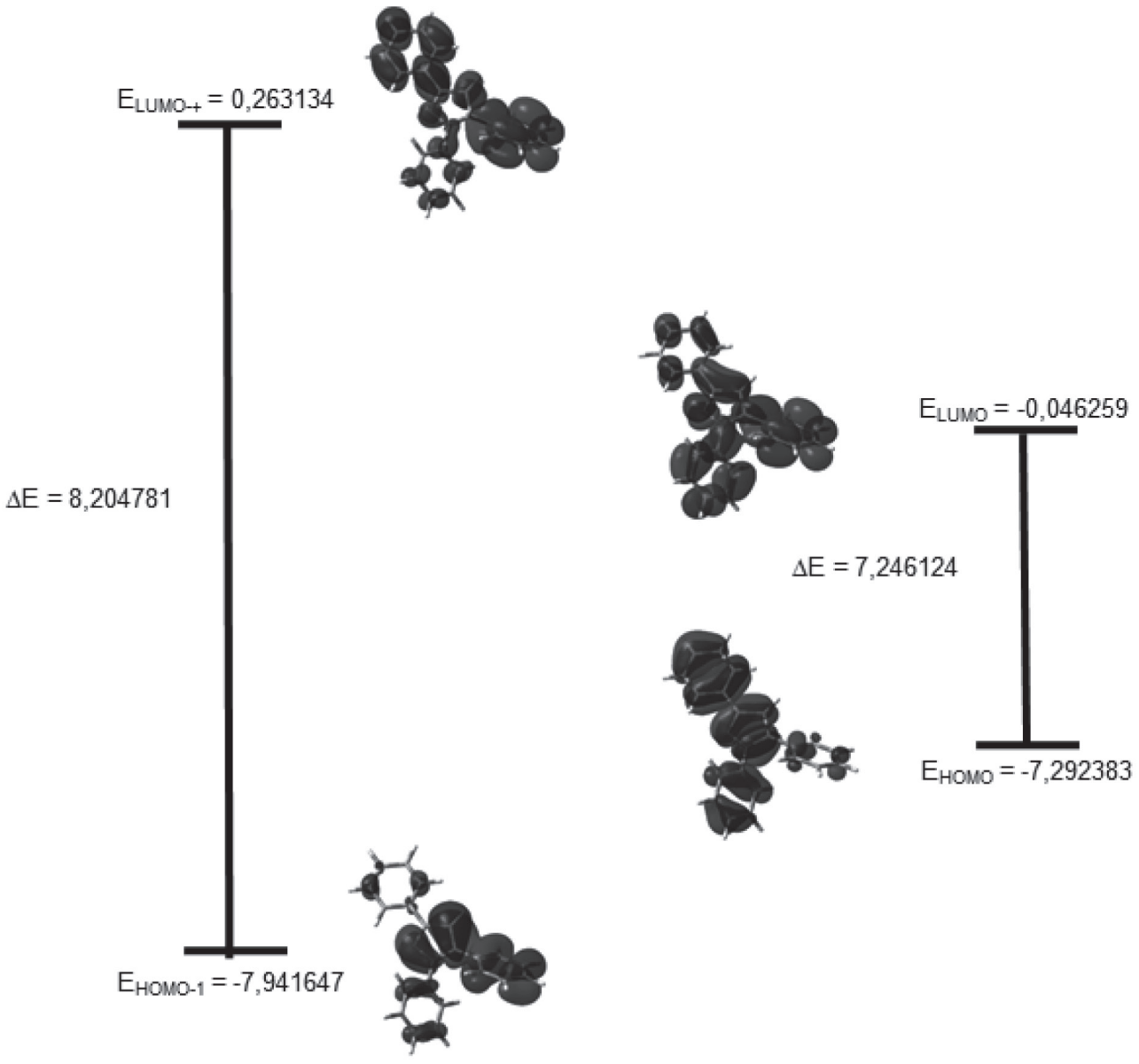

Figura 5. Representación gráfica del band-gap (HOMO) - (LUMO) para 1,3,5-trifenilpirazol.

Tabla 9. Valores calculados para los orbitales de frontera y su respectivo band-gap de 1,3,5-trifenilpirazol al nivel de cálculo DFT/cam-B-3LYP/6-311++G(d,p).

\begin{tabular}{lcc}
\hline \multicolumn{1}{c}{ Parámetro } & Unidad (au) & Unidad $(\mathrm{eV})$ \\
\hline LUMO & $-0,001700$ & $-0,046259$ \\
HOMO & $-0,267990$ & $-7,292383$ \\
Band-gap1 & 0,266290 & 7,246124 \\
LUMO+1 & 0,009670 & 0,263134 \\
HOMO-1 & $-0,291850$ & $-7,941647$ \\
Band-gap2 & 0,301520 & 8,204781 \\
\hline
\end{tabular}

Un band-gap de 7,246124 eV indica claramente que la molécula es muy estable, es decir, la transferencia de la carga está ocurriendo dentro de la molécula mediante el sistema espaciador. El compuesto en estudio no sería un buen candidato para ser utilizado como semiconductor, debido a este alto valor y posiblemente actuaria mejor como resistor [10]. En general los materiales semiconductores orgánicos incluyen hidrocarburos aromáticos policíclicos (HAP) donde las propiedades conductoras dependen de la brecha energética y está establecido que la energía de banda de separación de los aislantes es grande $(>4 \mathrm{eV})$, pero inferior para semiconductores $(<3 \mathrm{eV})$ [11]. En el diagrama también se muestra el band-gap entre los orbitales HOMO-1 y LUMO+1 de 8,204781 eV, lo cual indica para que se lleve a cabo una transición de electrones necesitaría mucha energía, al tener este un valor mayor que el bang-gap de los orbitales HOMO-LUMO.

A partir de las energías de los orbitales HOMO y se pueden determinar propiedades como potencial químico, electronegatividad, dureza y suavidad que describen la reactividad global de la molécula $[38,39]$. El potencial químico está relacionado con la electronegatividad, el cual indica que la densidad electrónica del sistema puede variar y los electrones pueden fluir de una región de alto potencial o mayor electronegatividad a uno 
de menor potencial o menor electronegatividad. El resultado obtenido para la molécula neutra $(-3,6596 \mathrm{eV})$ nos indica que la densidad electrónica del sistema puede variar espontáneamente, al tener este un valor negativo [40]. Por otro lado, el valor de electronegatividad de 3,6596 eV indica que la molécula tiende a atraer electrones sin cambiar su densidad electrónica. La dureza (n) corresponde a la separación entre el HOMO y LUMO, cuanto mayor es la brecha de energía orbital HOMO-LUMO, más dura es la molécula, lo que indica claramente que si la brecha de energía es mayor, la molécula será más dura, es decir, mide la resistencia impuesta por este al cambio en su distribución electrónica o polarizarse. Por tanto, el sistema, al tener un valor de dureza de $3,9708 \mathrm{eV}$ tendrá poca tendencia a dar o recibir electrones, es decir, la dureza se ha asociado con la estabilidad del sistema químico. En ese orden de ideas y aplicando la teoría de ácidos y bases de Parr y Pearson $[41,42]$, se tiene que el sistema en estudio posee un comportamiento de base dura, caracterizándose por su baja polarizabilidad, alta electronegatividad y por ser una especie donadora de electrones, por tanto, preferirá reaccionar con sistemas que presenten el comportamiento de un ácido duro. La suavidad puede medir el grado de reactividad química del compuesto y esta tiene un valor de 93,2391 eV y es el recíproco de la dureza [43].

Tabla 10. Descriptores de reactividad global de 1,3,5-trifenilpirazol.

\begin{tabular}{lcc}
\hline \multicolumn{1}{c}{ Parámetro } & Valor $(\mathrm{au})$ & Valor $(\mathrm{eV})$ \\
\hline Potencial de ionización $(I)$ & 0,2804 & 7,6303 \\
Afinidad electrónica $(A)$ & $-0,0114$ & $-0,3112$ \\
Potencial químico $(m)$ & $-0,1345$ & $-3,6596$ \\
Electronegatividad $(\mathrm{c})$ & 0,1345 & 3,6596 \\
Dureza $(h)$ & 0,1459 & 3,9708 \\
Suavidad $(S)$ & 3,4265 & 93,2391 \\
Electrofilicidad global & 0,0620 & 1,6864 \\
Band-gap & 0,2663 & 7,2461 \\
\hline
\end{tabular}

\section{Propiedades de óptica no lineal (ONL)}

Las componentes vectoriales del momento dipolar calculado fueron $\mu_{x}=-2,5736 \mathrm{D}, \mu_{\mathrm{y}}=-0,8183$ $\mathrm{D}$ y $\mu_{z}=0,0823 \mathrm{D}$ y el módulo es $2,702 \mathrm{D}$. La componente tiene la mayor contribución, debido a que ese es el eje que contiene los nitrógenos del anillo de pirazol. La polarizabilidad del sistema de electrones $\pi$ se logra uniendo grupos electro donadores y electro aceptores al principio y al final de los anillos o sistemas de anillos, con esto se crea un eje de transferencia de carga, por tanto, la polarizabilidad de una molécula es la medida de la capacidad de responder a un campo eléctrico y adquirir un momento eléctrico dipolar. Una sustitución en el fenilo en posición 5 permitiría lograr esta polarización.

Tabla 11. Momento dipolar $(\mu)$, polarizabilidad $(\alpha)$ e hiperpolarizabilidad $(\beta)$ de 1,3,5-trifenilpirazol, urea y $p$-nitroanilina al nivel de cálculo cam-B3LYP/6-311++G(d,p).

\begin{tabular}{cccc}
\hline Parámetro & $\mathbf{1 , 3 , 5 - t r i f e n i l p i r a z o l}$ & Urea & $\boldsymbol{p}$-nitroanilina \\
\hline$|\mu|$ & $2,702 \mathrm{D}$ & $3,973 \mathrm{D}$ & $7,210 \mathrm{D}$ \\
$\alpha_{\text {ave }}$ & $3,882 \times 10^{-23} \mathrm{esu}$ & $4,888 \times 10^{-24} \mathrm{esu}$ & $9,567 \times 10^{-24} \mathrm{esu}$ \\
$\Delta \alpha$ & $3,490 \times 10^{-23} \mathrm{esu}$ & $2,839 \times 10^{-24} \mathrm{esu}$ & $2,639 \times 10^{-23} \mathrm{esu}$ \\
$\beta_{\text {tot }}$ & $8,346 \times 10^{-31} \mathrm{esu}$ & $4,746 \times 10^{-31} \mathrm{esu}$ & $0,01176 \times 10^{-31} \mathrm{esu}$ \\
$\gamma_{\text {ave }}$ & $56,21 \times 10^{-36} \mathrm{esu}$ & $3,06 \times 10^{-36} \mathrm{esu}$ & $14,13 \times 10^{-36} \mathrm{esu}$ \\
\hline
\end{tabular}




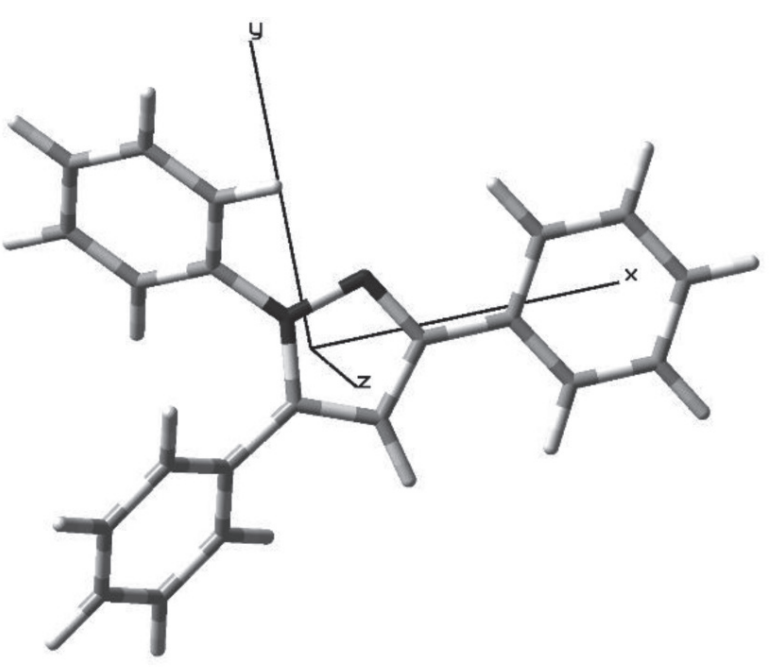

Figura 6. Distribución espacial de la molécula de 1,3,5-trifenilpirazol en los ejes cartesianos.

Los valores obtenidos para las polarizabilidades promedios se muestran en la Tabla 11, al igual que el valor de la anisotropía de la polarizabilidad (dependencia de la orientación molecular respecto a un campo eléctrico aplicado) en función de sus tensores (fuerza ejercida sobre una superficie de la molécula) sobre los ejes $x, y, z$. Los resultados de la polarizabilidad promedio muestran el comportamiento esperado en comparación con la urea y la $p$-nitroanilina. Se espera que por ser de mayor tamaño, sea mayor su valor. El valor de hiperpolarizabilidad $(\beta)$ (8,346 x 10-31 esu), 0,746 veces el valor para la urea y 709,6 el de la $p$-nitroanilina, muestra que el pirazol sería un material con buena respuesta óptica no lineal y posibles aplicaciones optoelectrónicas. Se sugiere que la deslocalización de electrones a lo largo del esqueleto conjugado y la asimetría de la molécula conducen a un aumento de la hiperpolarizabilidad molecular.

\section{Descriptores locales de reactividad}

Las funciones condensadas de Fukui para los estados aniónico $\left(f_{\mathrm{A}}^{-}\right)$, catiónico $\left(f_{\mathrm{A}}^{+}\right)$y radicalario $\left(f_{\mathrm{A}}{ }^{0}\right)$ se muestran en la tabla 12. Los valores de las funciones de Fukui negativos no se tendrán en cuenta, ya que no tienen significado físico, puesto que los valores de estas funciones están relacionados con la probabilidad de encontrar un electrón en un orbital, lo que corresponde al cuadrado de la función de onda del sistema, por tanto, nunca se obtendrán valores negativos. Observando los valores de $f^{+}$en la tabla 12 se tiene que los valores más altos $(0,1378-0,1319)$, los cuales corresponden a los hidrógenos de los fenilos, y les provocan características electrofílicas, lo que los hace susceptibles a un ataque nucleofílico. De igual forma, se puede analizar que el valor más alto de $f^{-}$lo posee el átomo de C15, con un valor de 0,2807 , lo que indica que este es el átomo más susceptible para el ataque de un electrófilo, debido a la alta densidad electrónica del fenilo al cual pertenece. El segundo valor más alto de $f$ corresponde al N1 del pirazol $(0,2700)$, como consecuencia al par electrónico solitario que posee. Siguiendo se tiene que el tercer valor más alto de $f^{-}$es el $\mathrm{C}_{7}$ con un valor de 0,2777 debido a la alta densidad electrónica del sistema fenil, que hace parte de este anillo, podrían experimentar reacciones de sustitución electrofílica aromática que llevarían a activar la posición para. Otro de los valores más altos de corresponde a $\mathrm{N}_{3}$, con un valor de 0,1975, este nitrógeno había presentado propiedades electrofílicas, pero comparando su $f^{+}$y $f^{-}$se encuentra que este último es mucho mayor, lo que indica que este nitrógeno tendrá mayor tendencia a reaccionar con electrófilos, ya que predomina la densidad del carga del sistema $\pi$ sobre el efecto inductivo.

Debido a que el sistema en estudio es grande, las funciones de Fukui tienden a diluirse entre los átomos y en muchos de los casos su diferencia presenta valores pequeños. Para estos casos se hace uso de otros descriptores locales de reactividad, llamados suavidades locales, los cuales complementan el estudio sobre los puntos más reactivos en la molécula. La suavidad local es directamente proporcional a la función de Fukui, por tanto, en este caso los valores más altos en los tres parámetros los poseen los mismos átomos. 
rev.ion. 2018;31(2):51-66. Bucaramanga (Colombia).

Tabla 12. Funciones de Fukui condensadas de 1,3,5-trifenilpirazol.

\begin{tabular}{|c|c|c|c|c|c|c|c|}
\hline \multirow[t]{2}{*}{ Átomo } & \multicolumn{3}{|c|}{$\begin{array}{l}\text { Función de Fukui } \\
\text { condensada }\end{array}$} & \multirow[t]{2}{*}{ Átomo } & \multicolumn{3}{|c|}{$\begin{array}{c}\text { Función de Fukui } \\
\text { condensada }\end{array}$} \\
\hline & $f^{-}$ & $f^{+}$ & $f^{0}$ & & $f^{-}$ & $f^{+}$ & $f^{0}$ \\
\hline N1 & 0,2700 & $-0,1158$ & 0,0771 & $\mathrm{C} 21$ & 0,1448 & $-0,1470$ & $-0,0011$ \\
\hline N2 & 0,2040 & $-0,2051$ & $-0,0006$ & $\mathrm{C} 22$ & 0,1207 & $-0,0950$ & 0,0129 \\
\hline $\mathrm{C} 3$ & 0,0422 & 0,0963 & 0,0693 & $\mathrm{C} 23$ & 0,1214 & $-0,1306$ & $-0,0046$ \\
\hline $\mathrm{C} 4$ & 0,2007 & $-0,1419$ & 0,0294 & $\mathrm{H} 24$ & $-0,1145$ & 0,1351 & 0,0103 \\
\hline C5 & $-0,0630$ & 0,0590 & $-0,0020$ & $\mathrm{H} 25$ & $-0,1296$ & 0,1378 & 0,0041 \\
\hline C6 & $-0,0875$ & 0,0539 & $-0,0168$ & $\mathrm{H} 26$ & $-0,1234$ & 0,1315 & 0,0040 \\
\hline $\mathrm{C} 7$ & 0,1555 & $-0,1299$ & 0,0128 & $\mathrm{H} 27$ & $-0,1121$ & 0,1327 & 0,0103 \\
\hline $\mathrm{C} 8$ & 0,1036 & $-0,0948$ & 0,0044 & $\mathrm{H} 28$ & $-0,1122$ & 0,1319 & 0,0098 \\
\hline $\mathrm{c9}$ & 0,1913 & $-0,1447$ & 0,0233 & $\mathrm{H} 29$ & $-0,1130$ & 0,1352 & 0,0111 \\
\hline C10 & 0,1029 & $-0,0973$ & 0,0028 & $\mathrm{H} 30$ & $-0,1099$ & 0,1199 & 0,0050 \\
\hline C11 & 0,1616 & $-0,1331$ & 0,0143 & H31 & $-0,1231$ & 0,1298 & 0,0034 \\
\hline $\mathrm{C} 12$ & 0,0745 & $-0,0486$ & 0,0130 & $\mathrm{H} 32$ & $-0,1055$ & 0,1283 & 0,0114 \\
\hline $\mathrm{C} 13$ & 0,1919 & $-0,1059$ & 0,0430 & $\mathrm{H} 33$ & $-0,1058$ & 0,1279 & 0,0111 \\
\hline $\mathrm{C} 14$ & 0,0946 & $-0,1131$ & $-0,0093$ & $\mathrm{H} 34$ & $-0,1098$ & 0,1295 & 0,0099 \\
\hline C15 & 0,2807 & $-0,1171$ & 0,0818 & H35 & $-0,1242$ & 0,1300 & 0,0029 \\
\hline C16 & 0,0841 & $-0,1062$ & $-0,0110$ & $\mathrm{H} 36$ & $-0,1219$ & 0,1321 & 0,0051 \\
\hline $\mathrm{C} 17$ & 0,1951 & $-0,1132$ & 0,0409 & $\mathrm{H} 37$ & $-0,1148$ & 0,1321 & 0,0087 \\
\hline C18 & 0,0225 & $-0,0477$ & $-0,0126$ & H38 & $-0,1140$ & 0,1326 & 0,0093 \\
\hline C19 & 0,1185 & $-0,1262$ & $-0,0038$ & H39 & $-0,1134$ & 0,1357 & 0,0111 \\
\hline $\mathrm{C} 20$ & 0,1168 & $-0,0982$ & 0,0093 & & & & \\
\hline
\end{tabular}

\section{Conclusiones}

En el presente trabajo, la estructura molecular optimizada del conformador estable, las propiedades vibratorias y electrónicas del 1,3,5-trifenilpirazol han sido calculadas por el método DFT/cam-B3LYP/6-311++G(d,p). Los parámetros geométricos optimizados (longitudes de enlace y ángulos de enlace) se determinan teóricamente y se comparan con los resultados experimentales. Las propiedades espectroscópicas de la presente molécula se examinaron mediante cálculos de frecuencia al mismo nivel y se compararon con los datos experimentales. Las asignaciones vibratorias de los números de onda se realizaron usando GausView 5. Las propiedades electrónicas también se calcularon con diferentes métodos para determinar el espectro UV-Vis. Las energías de $\mathrm{MO}$ y la $\lambda_{\max }$ del compuesto también se evalúan con el método TD-DFT/cam-B3LYP/6-311++G(d,p). Las energías HOMO y LUMO se calculan así como los valores de brecha de energía de los orbitales de frontera para una mejor comprensión de la transferencia de carga que podría ocurrir dentro de la molécula. El valor de hiperpolarizabilidad de primer orden del compuesto estudiado es 0,746 veces mayor que el valor de urea pero 709,6 veces mayor que para $p$-nitroanilina. Las funciones de Fukui mostraron que el anillo de fenilo en posición 1 y los átomos de nitrógeno son los sitios más probables para los ataques nucleofílicos y electrofílicos. El mapa MEP muestra que los sitios con potencial negativo son el átomo de nitrógeno en posición 2 y el fenilo en posición 3 .

\section{Referencias bibliográficas}

[1] Schlenker C, Barlier V, Chin S, Whited M, McAnally E, Forrest S, et al. Cascade Organic Solar Cells. Chem. Mater. 2011;23:4132-40.

[2] Morse G, Gantz J, Steirer K, Armstrong $\mathrm{N}$, Bender T. Pentafluorophenoxy Boron Subphthalocyanine $\left(\mathrm{F}_{5} \mathrm{BsubPc}\right)$ as a Multifunctional Material for Organic Photovoltaics. Appl. Mater. Interfaces. 
2014;6:1515-24.

[3] Ronchi M, Pizzotti M, Orbelli A, Righetto S, Ugo $\mathrm{R}$, Mussini $\mathrm{P}$, et al. Second-order nonlinear optical (NLO) properties of a multichromophoric system based on an ensemble of four organic NLO chromophores nanoorganized on a cyclotetrasiloxane architecture. J. Phys. Chem. C. 2009 ; 113: 2745-60.

[4] Andreu R, Garín J, Orduna J, Alcalá R, Villacampa B. Novel NLO-phores with proaromatic donor and acceptor groups. Org. Lett. 2003;5:3143-46.

[5] Ruiz M, Casado J, Hernández V, López J, Orduna J, Villacampa B, et al. Electronic, optical, and vibrational properties of bridged dithienylethylene-based NLO chromophores. J. Phys. Chem. C. 2008;112: 3109-20.

[6] Saravanan S, Balachandran V. Conformational stability, spectroscopic (FT-IR, FT-Raman and UV-Vis) analysis, NLO, NBO, FMO and Fukui function analysis of 4-hexylacetophenone by density functional theory. Spectrochim. Acta A. 2015;138:406-23.

[7] Demircioglu Z, Albayrak C, Büyükgüngör $O$. The spectroscopic (FT-IR, UV-vis), Fukui function, NLO, NBO, NPA and tautomerism effect analysis of (E)-2-[(2-hydroxy-6methoxybenzylidene)amino]benzonitrile. Spectrochim. Acta A. 2015;139:539-48.

[8] Gondek E. Photovoltaic solar cells based on pyrazole derivative. Mater. Lett. 2013;112:946.

[9] Amudha S, Austin S, Suthanthiraraj R, Maruthamuthu P. Performance characteristics of pyrazole as an effective dopant in a blended polymer electrolyte for nanocrystalline dyesensitized solar cell applications. Chem. Sci. Trans. 2013;2:S141-S146

[10] Ocaya R, Al-Sehemi G.A., Al-Ghamdi A, El-Tantawy F, Yakuphanoglu F. "Organic semiconductor photosensors". Journal of Alloys and Compounds, 2017;702:520-30.

[11] Costa J, Taveira R, Lima C, Mendes A, Santos L. Optical band gaps of organic semiconductor materials. Optical Materials, 2016;58:51-60.

[12]Akhtari K, Hassanzadeh K, Fakhraei B, Fakhraei N, Hassanzadeh $\mathrm{H}$, Zarei A. A density functional theory study of the reactivity descriptors and antioxidant behavior of Crocin. Comput. Theor. Chem. 2013;1013:123-29.

[13] Tathe A, Gupta V, Sekar N. Synthesis and combined experimental and computational investigations on spectroscopic and photophysical properties of red emitting 3-styryl coumarins. Dyes and Pigments. 2015;119:4955.

[14] Suvitha A, Periandy S, Gayathri P. Vibrational frequency analysis, FT-IR, FT-Raman, ab initio, HF and DFT studies, NBO, HOMOLUMO and electronic structure calculations on pycolinaldehyde oxime. Spectrochimica Acta A. 2014;117:216-24.

[15]Romani E, Brandán S. Structural and spectroscopic studies of two 1,3-benzothiazole tautomers with potential antimicrobial activity in different media. Prediction of their reactivities. Computational and Theoretical Chemistry, 2015;1061:89-99.

[16]Romani E, Ladetto M, Brandán S. Structural and vibrational studies of the potential anticancer agent, 5-difluoromethyl-1,3,4thiadiazole-2-amino by DFT calculations. Comput. Theor. Chem. 2013;1011:57-64.

[17]Kutsyna L, Korneeva O. The electronic structure of 1,3,5-triphenylpyrazole. J. App. Spec, 1971;15(2):1027-31.

[18] Akhtari K, Hassanzadeh K, Fakhraei B, Fakhraei N, Hassanzadeh H, Zarei S. A density functional theory study of the reactivity descriptors and antioxidant behavior of Crocin. Comput. Theor. Chem. 2013;1013:123-29.

[19] Nuñez F, Arguello E, Vivas R. Density functional study on electronic structures and reactivity in methyl-substituted chelates used in organic light-emitting diodes. Int. J. Quantum Chem, 2010;110(9):1622-36.

[20]Dennington R, Keith T, Millam J. GaussView, Version 5. Semichem Inc., Shawnee Mission, KS, (2009).

[21] Frisch M, Trucks G, Schlegel H, Scuseria G, Robb M, Cheeseman J, et. al. Gaussian 09, Revision A.02, Gaussian, Inc., Wallingford CT, 2009.

[22] Paschoal D, Dos Santos H. Assessing the quantum mechanical level of theory for prediction of linear and nonlinear optical properties of push-pull organic molecules. J. Mol. Mod. 2013;19:2079-90.

[23] Wazzan N, Al-Qurashi O, Faidallah H. DFT/ and TD-DFT/PCM calculations of molecular structure, spectroscopic characterization, NLO and NBO analyses of 4-(4-chlorophenyl) and 4-[4-(dimethylamino) phenyl]-2-oxo-1,2,5,6tetrahydrobenzo[H]quinoline-3-carbonitrile dyes. J. Mol. Liq. 2016;223:29-47.

[24] Gil D, Defonsi M, Estévez-Hernández O, 
Duque J, Reguera E. Quantum chemical studies on molecular structure, spectroscopic (IR, Raman, UV-Vis), NBO and HOMO-LUMO analysis of 1-benzyl-3-(2-furoyl) thiourea, Spectrochim. Acta A. 2015;145:553-62.

[25] Sundaraganesan N, Ilakiamani S, Saleem H, Wojciechowski P, Michalska D. FT-Raman and FT-IR spectra, vibrational assignments and density functional studies of 5-bromo2-nitropyridine, Spectrochim. Acta A. 2005;61:2995-3001.

[26] CambridgeSoft. PerkinElmer. Versión 13.0.0.3015. 1996-2012.

[27] Mestrelab Research S.L. Version 60.2.-5475. 2009.

[28] Trotter J. Bond lengths in benzene derivatives: Hybridization or resonance. Tetrahedron. 1960;8:13-22.

[29] Shetty M, Samant S. Sulfamic Acid $\left(\mathrm{H}_{2} \mathrm{NSO}_{3} \mathrm{H}\right)$ : A low-cost, mild, and efficient catalyst for the synthesis of substituted N-Phenylpyrazoles under solvent-free conditions. Synthetic Commun. 2012;42:1411-18.

[30] Sharma Y. Elementary Organic Spectroscopy, principles and chemical applications. India: Chande \& Company Ltd.; 1994.

[31] Krishnakumar V, Manohar S, Nagalakshmi $R$. Crystal growth and characterization of $\mathrm{N}$-hydroxyphthalimide $\left(\mathrm{C}_{8} \mathrm{H}_{5} \mathrm{NO}_{3}\right)$ crystal. Spectrochim. Acta A. 2008;71:110-5.

[32] Ananthnag G, Adhikari A, Balakrishna M. Ironcatalyzed aerobic oxidative aromatization of 1,3,5-trisubstituted pyrazolines. Catal. Commun. 2014;43:240-3.

[33] Nakamichi N, Kawashita $Y$, Hayashi $M$. Oxidative aromatization of 1,3,5-Trisubstituted pyrazolines and hantzsch 1,4-dihydropyridines by $\mathrm{Pd} / \mathrm{C}$ in acetic acid. Org. Lett. 2002;4(22):3955-7.

[34]Han B, Liu Z, Liu Q, Yang L, Liu Z-L, Yu W.
An efficient aerobic oxidative aromatization of Hantzsch 1,4-dihydropyridines and 1,3,5-trisubstituted pyrazolines. Tetrahedron. 2006;62(11):2492-96.

[35] Carrillo J, Cossío, F, Díaz-Ortiz A, GómezEscalonilla M, Begoña A, Moreno A, Prieto P. A complete model for the prediction of ${ }^{1} \mathrm{H}$ - and ${ }^{13} \mathrm{C}$-NMR chemical shifts and torsional angles in phenyl-substituted pyrazoles". Tetrahedron. 2001;57:4179-87.

[36] Begtrup $M$, Vedsù $P$, Cabildo $P$, Claramunt $\mathrm{RM}$, Elguero J, Meutermans W. ${ }^{13} \mathrm{C}$ NMR of pyrazoles. Magn. Reson. Chem. 1992;30:10768.

[37] Ando W, Sato R, Yamashita M, Akasaka T, Miyazaki $H$. Quenching of singlet oxygen by 1,3,5-triaryl-2-pyrazolines. J. Org. Chem. 1983;48:542-6.

[38] Arjunan V, Balamourougane $P$, Kalaivani $M$, Raj A, Mohan S. Experimental and theoretical quantum chemical investigations of 8-hydroxy5-nitroquinoline. Spectrochim. Acta A. 2012;96:506-16.

[39] Fukui K. Role of frontier orbitals in chemical reactions. Science. 1982;218:747-54.

[40]López J, Ensuncho A, Robles J. Estudio teórico de la reactividad química y biológica de cisplatino y algunos derivados con actividad anticancerosa. Información Tecnológica. 2013;24(3):3-14.

[41] Pearson R. Hard and soft acids and Basis. J. Am. Chem. Soc. 1963;85(22):3533-39.

[42] Parr R, Pearson R. Absolute hardness: companion parameter to absolute electronegativity. J. Am. Chem. Soc. 1983;105(26):7512-16.

[43] Chandrasekaran K, Kumar R. Structural, spectral, thermodynamical, NLO, HOMO, LUMO and NBO analysis of fluconazole. Spectrochim. Acta A. 2015;150:974-91. 